\title{
A qualitative estimation of the influence of transport factors on the work efficiency
}

\author{
Alexandru Ene ${ }^{1, *}$ and Daniel-Constantin Anghel ${ }^{2}$ \\ ${ }^{1}$ University of Pitesti, Department of Electronics, Computers, Communications and Electrical \\ Engineering, Str. Tg. Din Vale, nr. 1, Pitesti, Romania \\ ${ }^{2}$ University of Pitesti, Department of Manufacturing and Industrial Management, Str. Tg. Din Vale, \\ nr. 1, Pitesti, Romania
}

\begin{abstract}
In this paper we make a qualitative estimation of the influence of transport factors on the work efficiency (labour yield). There will be three classes of influence: positive influence, no influence and negative influence. We take into account some transportation factors like the level of noise during the transport, the level of temperature (the thermic comfort), etc. We use a software simulated feed forward neural network in order to solve this classification problem. The network will divide the input data (values for the transport factors) into these three classes. The input data are stored in an XML file, and they are obtained from a questionnaire that was completed by people working in automotive industry. The results are both useful for the transport companies and for the industry managers.
\end{abstract}

\section{Introduction}

Work efficiency is primarily a function of an individual's effort to obtain the desired outcomes. But is also widely accepted that work efficiency is multidimensional [1]. It is usual in our days for many employed people to come to their work from their homes that are located at considerable distance, using buses or other cars from transport companies. Transport factors do influence these people work efficiency, either in a positive way or in a negative way. So, both industrial enterprises and transport companies are interested that these workers to have travel conditions that will increase their work efficiency.

In order to determine the way in which transport factors influence the labour yield, we collected data using a questionnaire, for the workers from an enterprise, workers that come to work from distant locations, using buses from a transport company.

We took into account the following nine transport factors:

1. The duration of the journey

2. The position of the person while traveling

3. The presence/ absence of road abnormalities

4. The thermal comfort

5. The acoustic comfort

6. The level of air pollution

7. The humidity level

\footnotetext{
${ }^{*}$ Corresponding author: daniel.anghel@upit.ro
} 
8. The driving style of the driver

9. The number of stops on the route

Each input factor could have five different input values from low to high. In our questionnaire these values were coded with: -2 (corresponds to low), -1, 0, 1, 2 (corresponds to high). For a set of nine input factor values, was recorded as an output value, the influence of these particular transport factors on the labour yield. The output value could be: negative influence, no influence or positive influence ( $-1,0$ or 1$)$.

We collected based on the questionnaire some tens of sets of data.

The data generated from the questionnaire, were stored in an XML computer file.

XML is a mark-up language as HTML. While HTML describes the way in which data are displayed by the browser on the monitor using predefined tags, XML describes the structure of data, using tags that are built by the designer. XML files are widely used for data transmission in Internet.

A typical record from the $\mathrm{XML}$ file is:

$<$ record $>$

$<$ duration $>1<$ duration $>$

$<$ position $>2<$ /position $>$

$<$ road $>1</$ road $>$

$<$ temperature $>1</$ temperature $>$

$<$ noise $>1</$ noise $>$

$<$ pollution $>0<$ /pollution $>$

$<$ humidity $>0<$ /humidity $>$

$<$ driver $>1<$ driver $>$

$<$ stops $>1</$ stops $>$

$<$ efficiency $>-1</$ efficiency $>$

$<$ record $>$

A record has ten fields: the first nine fields are for the input transport factors, and the tenth field is the work efficiency, i.e. the output influenced by all transport factors.

Because each input parameter can have five different values, and there are nine input parameters, there are $5^{9}=1953125$ different possible input combinations. Of course, we cannot have a questionnaire that will cover all these combinations but, we want to be able to recognize from any possible input combination of transport factors, how this will influence the work efficiency. For this task, we used a feed forward neural network that is able to learn patterns and to generalize, recognizing new patterns, which it never learned. Neural networks have the ability to recognize hidden patterns, hidden relationships between variables and that is why they are adequate tools for processing the results of a questionnaire in order to make future output predictions when new input data are available. Our study of the influence of transport factors on work efficiency is an application of neural networks, but it uses a new way in which our input data are pre-processed (binary coded) in order to be applied on the inputs of the neural network.

\section{Feed forward neural networks}

Multilayer perceptron (MLP) also called feed forward neural networks consist of layers of interconnected artificial neurons (processing units). They are nonlinear devices that are able to learn to recognize patterns using learning examples. So, they are widely used in pattern recognition applications in industry, finance, medicine, military and other fields.

In order to recognize an object in an image, a MLP network does not need a sophisticated mathematical model of the object to be recognized. The network is trained to recognize the object, by presenting it different images of that object (these are called training patterns), using the backpropagation learning algorithm. Through this algorithm, the weights of the 
connections between the neurons are calculated. These weights quantify the strength of connections.

This is a supervised learning algorithm and it starts initializing all the weights with small positive and negative real values, random generated.

The pseudo code of backpropagation algorithm is [2]:

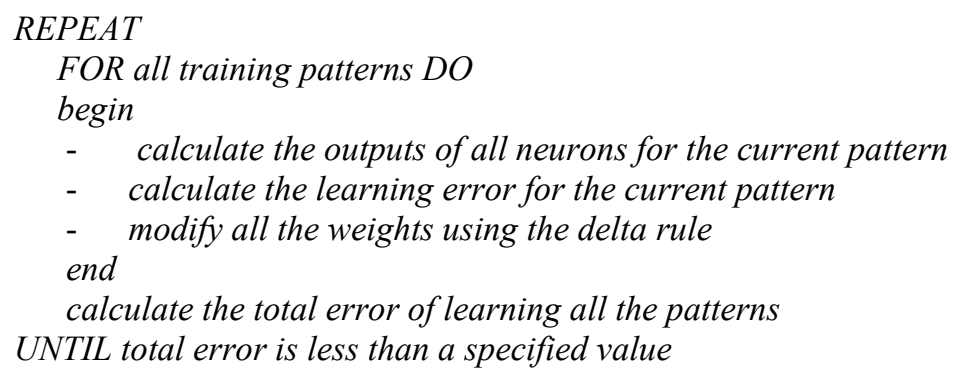

When all training patterns had been presented to the network and the weights had been modified according to the backpropagation algorithm, it is said that there was a training epoch.

Usually, for a pattern recognition application, a feed forward neural network needs to have three neuronal layers: an input layer, a hidden layer and an output layer. Each neuron from a layer is connected with all neurons from the next layer, as is illustrated in Figure 1.

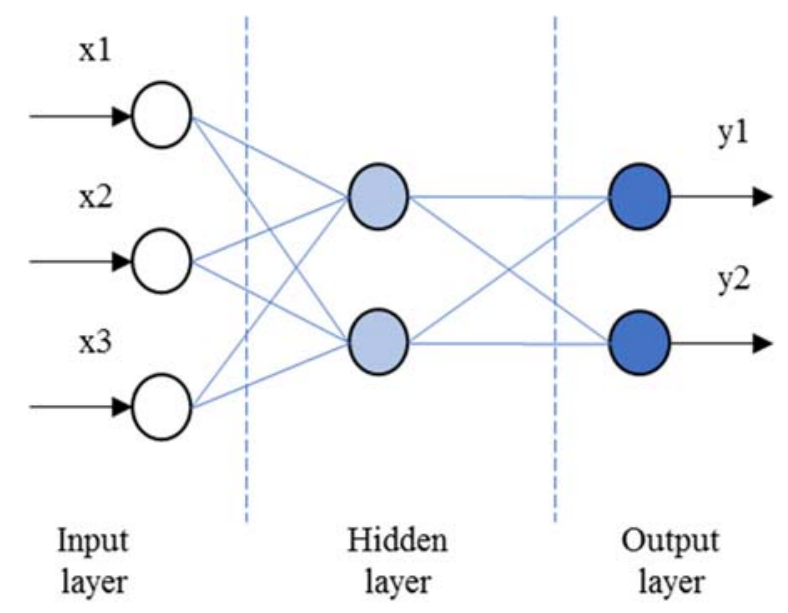

Fig. 1. A feed forward neural network (3-2-2).

The number of neurons from input layer and from the output layer is determined from the problem that has to be solved by the neural network. The number of neurons from the hidden layer is chosen experimentally.

The weights of the connections between neurons are calculated by the backpropagation algorithm, based on the training patterns. Typically, these weights are saved in a computer file.

After the network was trained, it can be used to recognize other patterns.

In [3] we used a feed forward neural network to estimate the quality of a working place, a typical application in ergonomics. 


\section{The Java application}

The feed forward neural network was simulated using a Java software application. We used a $45-14-3$ neural network architecture. We used 5 input neurons because we have 9 input parameters, each parameter having 5 possible different values. Neural networks recognize better when they have binary inputs instead of analog inputs. We had for each input parameter 5 possible analog values, as obtained from the questionnaire: $-2,-1,0,1$ and 2 . We binary coded these analog values, in the following manner:

10000 for analog value -2

01000 for analog value -1

00100 for analog value 0

00010 for analog value +1

00001 for analog value +2

So, we used 5 bits for a single input parameter, so, we have $9 \times 5=45$ input neurons.

The network estimates based on the 9 input parameters the work efficiency, which can have 3 different possible values, and, that is why we use 3 output neurons (each output could be 0 or 1), with the following codification:

\section{0 - negative influence on work efficiency}

010 - no influence on work efficiency

o 01 - positive influence on work efficiency

The training patterns are obtained from the XML file and are saved in a text file, on the disk. Here is an example of a training pattern.

000100000100010000100001000100001000001000010 100

The Java application trains the feed forward neural network using the training patterns stored in the text file. It also saves the weights of the trained network in a text file. The software application also uses the neural network to estimate the influence on the work efficiency of any possible combination of input parameters that are presented on the inputs of the network.

\section{The simulation results}

The neural network was trained to learn the training patterns with a $1 \%$ learning error. It converged typically in less than 200 epochs (this was determined by the number of the training patterns that were available for the training).

In order to test the network's ability to generalize, we tested it with 4 different sets of input data. For these tests we knew from the questionnaire which is the influence of the input transport factors on the work efficiency. The neural network gave correct answers in all tests, as illustrated by the following execution of the program:

Epoch $1:$ total error $=3.9180432909401643$

Epoch 2 : total error $=4.062531934847529$

Epoch $155:$ total error $=0.010055073320472785$

Epoch $156:$ total error $=0.009973958266715164$ 
The network converted successfully!

The weights had been saved successfully!

test 1

Ideal value: 1 Neural network calculated value: 0.9619412871122635

Ideal value: 0 Neural network calculated value: 0.021876857834777146

Ideal value: 0 Neural network calculated value: 0.03611935020568179

test $1: O K$ !

test 2

Ideal value: 0 Neural network calculated value: 0.01878252776436474

Ideal value: 1 Neural network calculated value: 0.9768090371862408

Ideal value: 0 Neural network calculated value: 0.015448165093419076

test $2: O K$ !

test 3

Ideal value: 0 Neural network calculated value: 0.02088317236801642

Ideal value: 0 Neural network calculated value: 0.006756006112959953

Ideal value: 1 Neural network calculated value: 0.9861565373784594

test $3: O K$ !

test 4

Ideal value: 0 Neural network calculated value: 0.06799214508178054

Ideal value: 0 Neural network calculated value: 0.003515613938580661

Ideal value: 1 Neural network calculated value: 0.9770592179178614

test $4: O K$ !

Total number of correct answers $=4$ from 4

\section{Conclusions}

In this paper, we used a neural network, to make a qualitative estimation of the influence of nine transport factors on the work efficiency. The necessary data for training and for testing the neural network were obtained from a questionnaire. The neural network was simulated using a Java software application, and it learned very well the training examples and also answered correctly to the tests with input data that had not been used for the training. So, the neural network is able to generalize.

The correctness of the answers offered by the network very much depends on the dimension and on the quality of the training patterns. The results obtained from the neural network are useful both to transport companies and industrial enterprises.

\section{References}

1. S. A. Stumpf, S. Rabinowitz, Journal of Vocational Behaviour, 18(2), 202 (1981)

2. A. Ene, C. Stirbu, Neural Networks. Theory and applications in Java (in Romanian), (University of Pitesti Publishing House, Romania, 2008)

3. A. Ene, D. C. Anghel, (2013, June). A neural networks application in ergonomics, Electronics, Computers and Artificial Intelligence, ECAI, IEEE, 1, (2013) 